Universidad de La Salle

Ciencia Unisalle

2020-12-01

\title{
Evaluación de la adaptación de fríjol rojo cuarentano (Phaseolus vulgaris) para producción de grano seco en las condiciones de El Yopal, Casanare
}

Duber Alejandro Pinto

Universidad de La Salle, Bogotá

Diana Katherine Rios

Universidad de La Salle, Bogotá

Follow this and additional works at: https://ciencia.lasalle.edu.co/utopia_working_papers

\section{Recommended Citation}

Pinto, Duber Alejandro and Rios, Diana Katherine, "Evaluación de la adaptación de fríjol rojo cuarentano (Phaseolus vulgaris) para producción de grano seco en las condiciones de El Yopal, Casanare" (2020).

Utopía - Working papers. 7.

https://ciencia.lasalle.edu.co/utopia_working_papers/7

This Documento de trabajo is brought to you for free and open access by the Utopía at Ciencia Unisalle. It has been accepted for inclusion in Utopía - Working papers by an authorized administrator of Ciencia Unisalle. For more information, please contact ciencia@lasalle.edu.co. 
https://doi.org/10.19052/wp.utopia.2020.3

\title{
Utopía | 10 \\ Working Papers \\ 2020
}

\section{Evaluación de la adaptación de fríjol rojo cuarentano (Phaseolus vulgaris) para producción de grano seco en las condiciones de El Yopal, Casanare}

\author{
Duber Alejandro Pinto ${ }^{1}$, Diana Katherine Rios ${ }^{2}$
}

\begin{abstract}
RESUMEN
El fríjol cuarentano rojo (Phaseolus vulgaris), es un cultivo de ciclo corto, cultivado principalmente en la región caribe de Colombia como cultivo de pan coger. La especie posee un buen contenido de proteínas, es muy tolerante a la sequía y por ser una leguminosa, fija nitrógeno al suelo. Los principales cultivos en El Yopal son la palma africana (Elaeis guineensis) y el arroz (Oriza sativa), otra actividad económica relevante es la producción de ganado bovino. Sin embargo, hay una limitada oferta agrícola de otros cultivos, que potencialmente se pueden adaptar a estas condiciones. De esta manera, se evaluó el efecto de tres densidades de siembra del fríjol rojo cuarentano bajo las condiciones ambientales de Yopal. El cultivo tuvo registro como componentes de rendimiento un número de vainas por planta 38,05 unidades, con una longitud de 17,15 cm. Las semillas por vaina fueron 15,33, el peso de 100 semillas fue de 16,09 gramos y un rendimiento por tratamiento de 7,6 y $5 \mathrm{~kg}$ respectivamente por unidad de área. Los resultados indican que esta especie vegetal puede ser cultivada en El Yopal y convertirse en una alternativa alimenticia para pequeños productores.
\end{abstract}

Palabras clave: Seguridad alimentaria, Leguminosas, Nutrición

Evaluation of the adaptation of red beans cuarentano (Phaseolus vulgaris) for dry grain production under conditions of Yopal, Casanare

\begin{abstract}
The red beans cuarentano (Phaseolus vulgaris) is a short-cycle crop, planted mainly in the Caribbean region of Colombia as a subsistence crop. The species has a good protein content, is very tolerant to drought and, being a legume, fixes nitrogen to the soil. The main crops in El Yopal are the African palm (Elaeis guineensis) and rice (Oriza sativa), another relevant economic activity is the production of cattle. However, there is a limited agricultural supply of other crops, which can potentially be adapted to your ambiental conditions. In this way, the effect of three planting densities of red beans cuarentano was evaluated under the environmental conditions of Yopal. The crop registered as yield components, 38.05 pods per plant, with a length of $17.15 \mathrm{~cm}$. The seeds per pod were 15.33, the weight of 100 seeds was 16.09 grams and a yield per treatment of 7, 6 and $5 \mathrm{~kg}$ respectively per unit area. The results indicate that this plant species can be cultivated in El Yopal and become a food alternative for small producers.
\end{abstract}

Keywords: Food safety, Legumes, Nutrition

\footnotetext{
${ }^{1}$ Estudiante de ingeniería Agronómica de la Universidad de la Salle

${ }^{2}$ Docente del programa de ingeniería Agronómica de la Universidad de La Salle
} 


\section{Utopía | \\ Working Papers \\ 2020}

\section{INTRODUCCIÓN}

El agro colombiano presenta debilidades como una lenta transferencia tecnológica, bajos niveles de instrucción para los campesinos, calidad de los suelos, baja inversión en tecnología e infraestructura vial deficiente (Giral, 2016), en este sentido, se ha venido generando una crisis para el sector agrícola generada por los altibajos en los precios en el momento de la venta de la cosecha. Por ende, los ingresos de muchos cultivadores, especialmente de los más pequeños, no alcanzan para cubrir los costos de producción. Lo que significa que miles de campesinos trabajan a pérdida o apenas para sobrevivir, sea obteniendo lo básico para adquirir la canasta familiar o dependiendo de ciertos cultivos para su alimentación (Tovar, 2013).

El fríjol rojo cuarentano es una especie vegetal de ciclo corto, plantado principalmente en la región caribe de Colombia por campesinos como cultivo de pancoger. La especie posee unas características que favorecen su cultivo, así como un buen contenido de proteínas, es tolerante a la sequía, y por ser una leguminosa fija nitrógeno al suelo, aspecto importante en donde los suelos son poco fértiles (Consuegra, 2018).

Las actividades agrícolas de El Yopal, Casanare son el cultivo de palma de aceite (Elaeis guineensis) y arroz (Oryza sativa). También se destaca como actividad rural la ganadería (bovinos). Desde esta perspectiva, se propuso una investigación que buscaba evaluar el comportamiento del fríjol rojo cuarentano, en una zona donde las características ambientales son bastante contrastantes, pues posee un periodo de abundantes lluvias y un periodo prolongado de sequía. Están son algunas de las variables ambientales que un cultivo se debe enfrentar en El Yopal, Casanare. Adicionalmente, debe diversificarse la actividad económica rural, pues está se centra en la ganadería principalmente. De esta manera, si esta variedad de frijol se adapta a las condiciones ambientales tendrá un alto potencial de ser una verdadera alternativa, tanto de alimentación local, como para su comercialización y/o transformación de la materia prima. 


\section{Utopía | \\ Working Papers \\ 2020}

\section{METODOLOGÍA}

El experimento fue realizado en La Universidad de La Salle Campus Utopía (5 19'91.11” N y 72 1'719.30" O), en la línea productiva de cereales y leguminosas.

Se utilizó un diseño de bloques completos al azar (BCA) con cuatro bloques y tres tratamientos por bloque. El área total utilizada fue de $400 \mathrm{~m}^{2}$ ( $16 \mathrm{~m}$ de ancho y $25 \mathrm{~m}$ de largo), con parcelas de 5,5m de largo y $4 \mathrm{~m}$ de ancho $\left(22 \mathrm{~m}^{2}\right)$. En cada bloque se distribuyeron aleatoriamente tres tratamientos para un total de 12 unidades experimentales (parcelas).

Cada unidad experimental constó de 4 surcos, con una distancia entre surco de $1 \mathrm{~m}$ y entre plantas (0,3. 0,4 y 0,5 m), obteniendo tres diferentes densidades de siembra. De esta manera, el área útil quedó constituida por los dos surcos centrales en cada densidad de siembra evaluada. La distancia entre parcelas y bloques fue de $1 \mathrm{~m}$. Se utilizaron 2.068 semillas en el área total semillas, las cuales germinaron 3 a 4 días después de la siembra (DDS). Al realizar una extrapolación de cada densidad de siembra evaluada, se encuentra que se necesitarían $14.2 \mathrm{~kg} / \mathrm{ha}^{-1}$ en la primera densidad (A) (1 x 0,3 m); $11.7 \mathrm{~kg} / \mathrm{ha}^{-1}$ en la segunda densidad (B) (1 x 0,4 m) y 9.36 $\mathrm{kg} / \mathrm{ha}^{-1}$ en la tercera densidad (C) (1 x 0,5 m). De esta manera se obtendrían el siguiente número de plantas: $33.333 / \mathrm{ha}^{-1}$ para la primera densidad, 25.000/ha $\mathrm{h}^{-1}$ para la segunda densidad, y 20.000/ha ${ }^{-1}$ para la tercera densidad. Las semillas fueron sembradas el día 24 de julio de 2019 y la cosecha se recolecto 75 DDS.

Durante el desarrollo del cultivo, se realizaron labores culturales como: fertilización, la cual fue hecha con base en un plan de fertilización elaborado para el cultivo teniendo como referente la cantidad extracción de nutrientes por hectárea y la disponibilidad de estos presentes en el suelo según el correspondiente análisis de suelo del lote donde se estableció el ensayo. Como fuentes de elemento mayores se usaron, Urea (46-0-0), DAP (18-46-0) y $\mathrm{KCl}$ (0-0-60). La fertilización fue edáfica, en forma de corona a unos $10 \mathrm{~cm}$ de la raíz de la plántula, y posterior a ello se realizó el tapado del fertilizante para evitar pérdidas por la alta precipitación del mes de julio. En total por planta se le aplicaron 7,8 gramos. 
https://doi.org/10.19052/wp.utopia.2020.3

\section{Utopía | \\ Working Papers \\ 2020}

El manejo de arvenses se realizó de forma manual al momento de realizar la fertilización. De esta manera se máximo el esfuerzo físico. Entretanto, el manejo de plagas y enfermedades se efectuó a partir me monitoreos, los cuales tuvieron por objetivo: conocer el estado sanitario del cultivo, ver el estado de evolución de las plagas y enfermedades, y controlar la efectividad de las medidas utilizadas para el control de alguna plaga o enfermedad (Mitidieri \& Polack, 2012). El monitoreo se realizó en un metro lineal de cultivo, revisando las plantas presentes para evidenciar plagas o enfermedades.

Las variables de rendimiento evaluadas fueron: número de vainas por planta, longitud de las vainas, semillas por vaina, peso de 100 semillas y producción por densidad de siembra. Las variables fueron sometidas a Análisis de Varianza (Anova) y a prueba posthoc de Tukey $(\alpha=0,05)$, cuando fue necesario. Los Análisis fueron realizados en el programa estadístico InfoStat.

\section{RESULTADOS Y DISCUSIÓN}

Para la variable número de vainas por planta, no se presentó diferencia significativa entre las densidades de siembra, reportando un número promedio general de vainas por planta de 38,05 vainas (figura 1).

Para la variable longitud de vainas, no se presentó diferencia significativa entre las densidades de siembra, reportando un tamaño promedio general de vaina de $17,15 \mathrm{~cm}$ (figura 2).

Para la variable número de semillas por vaina, no se presentó diferencia significativa entre las densidades de siembra, reportando un número promedio general por vaina de 15,33 semillas (figura 3). 


\section{Utopía | \\ Working Papers \\ 2020}

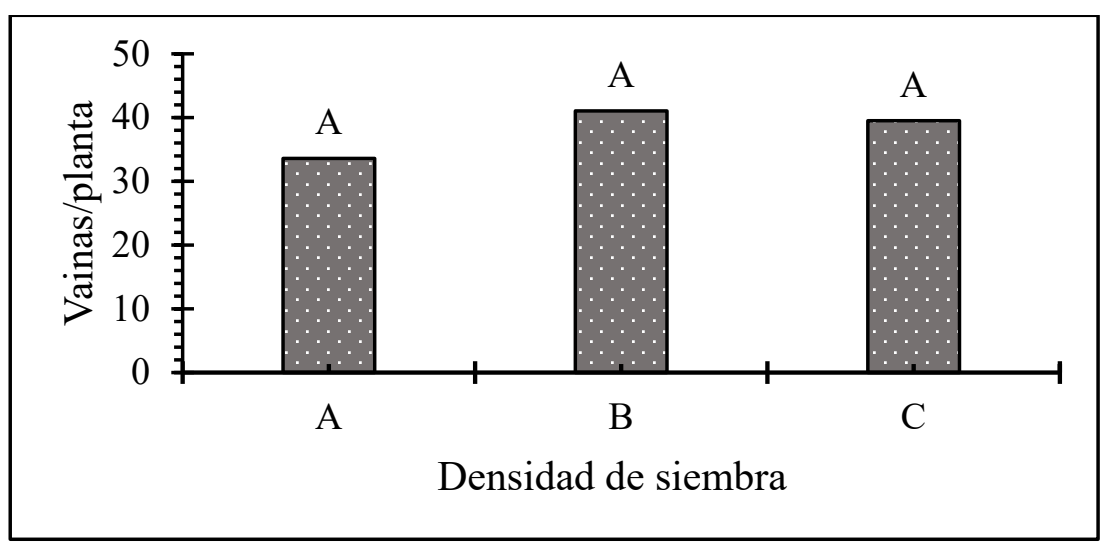

Figura 1. Comparación de la diferencia mínima significativa $(\mathrm{DMS}=22,22)$, entre las diferentes densidades de siembra evaluadas para la variable número de vainas por planta.

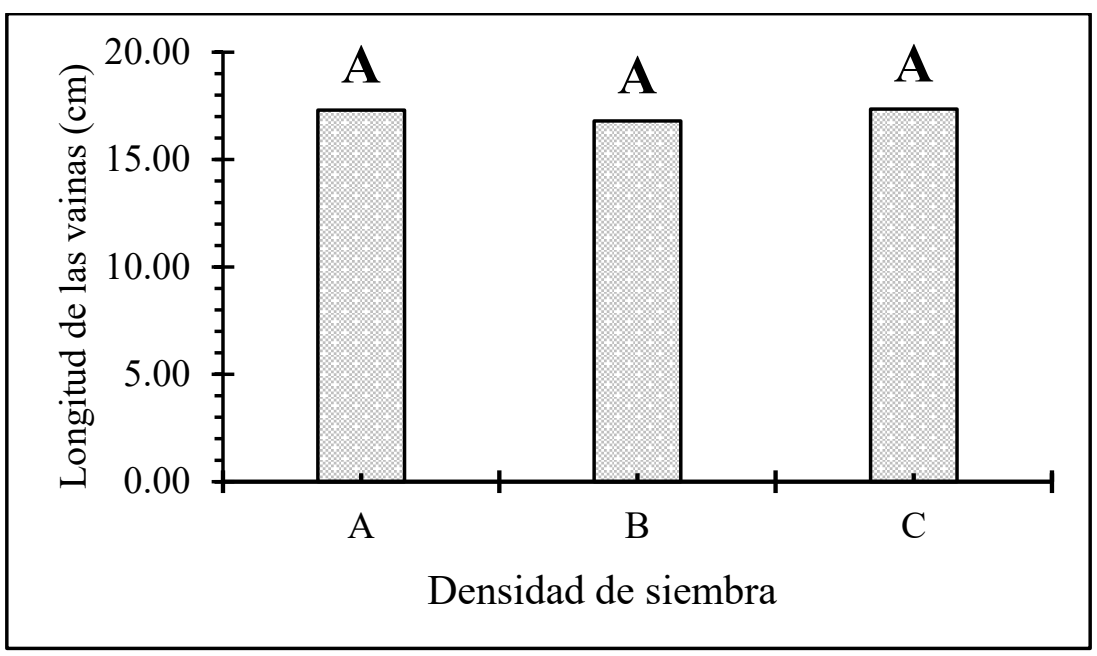

Figura 2. Comparación de la diferencia mínima significativa $(\mathrm{DMS}=2,46)$, entre las diferentes densidades de siembra evaluadas para la variable longitud de las vainas. 


\section{Utopía | \\ Working Papers \\ 2020}

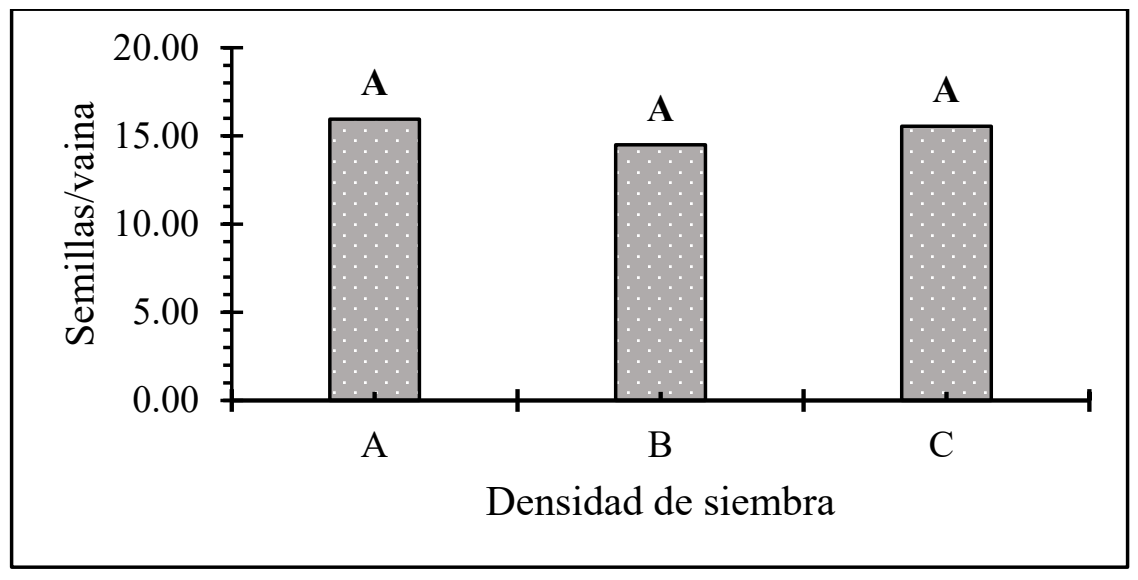

Figura 3. Comparación de la diferencia mínima significativa $(\mathrm{DMS}=1,75)$, entre las diferentes densidades de siembra evaluadas para la variable la variable número de semillas por vaina.

Para la variable peso de 100 semillas, no se presentó diferencia significativa entre las densidades de siembra, reportando un peso promedio general de 100 semillas de 16,09 gramos (figura 4).

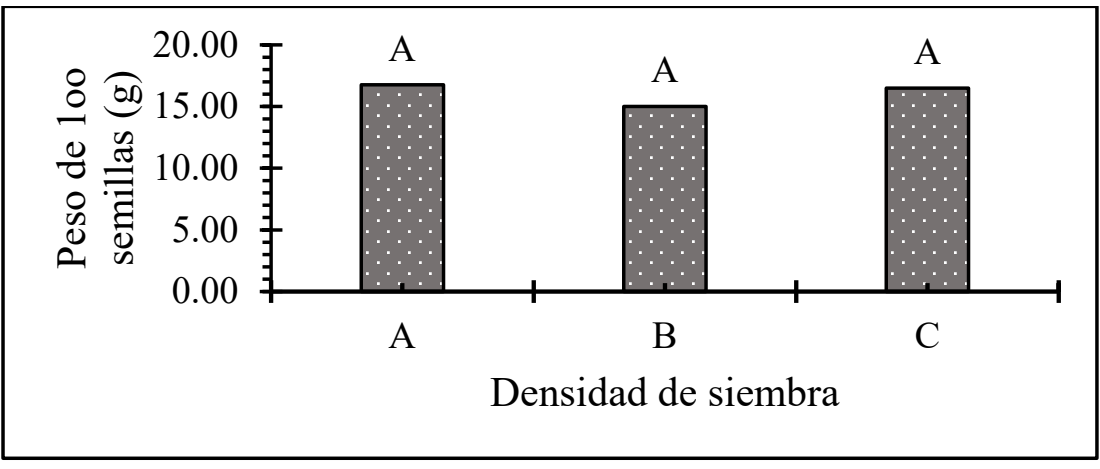

Figura 4. Comparación de la diferencia mínima significativa (DMS = 2,59), entre las diferentes densidades de siembra evaluadas para la variable peso de 100 semillas.

Entretanto, la variable producción presento diferencias significativas entre las densidades de siembra evaluadas. La densidad de 1 x 0,3 metros fue superior a las otras dos evaluadas y presento un total de $7 \mathrm{~kg}$ por unidad de área. 


\section{Utopía | \\ Working Papers \\ 2020}

Finalmente, los costos de producción por hectárea para la obtención de grano seco de fríjol rojo cuarentano son presentados en la tabla 1 .

Tabla 1. Costos de producción para una hectárea de fríjol rojo cuarentano, en las condiciones de El Yopal, Casanare.

\begin{tabular}{|c|cc|}
\hline \multirow{2}{*}{$\begin{array}{c}\text { Interpretación de utilidad según el uso al fríjol rojo cuarentano } \\
\text { en }\left(1 \mathrm{ha}^{-1}\right) \text { en Yopal Casanare. }\end{array}$} \\
\hline \multirow{2}{*}{$\begin{array}{c}\text { Descripción } \\
\text { Uso }\end{array}$} \\
\hline Mano de obra & $\$$ & $2.100 .000,00$ \\
\hline Insumos & $\$$ & $807.000,00$ \\
\hline Materiales y herramientas & $\$$ & $185.000,00$ \\
\hline Fletes/transporte & $\$$ & $120.000,00$ \\
\hline Costos indirectos & $\$$ & $135.850,00$ \\
\hline Total de inversión & $\mathbf{3 . 3 4 7 . 8 5 0 , 0 0}$ \\
\hline \multicolumn{2}{|c|}{ Índices financieros } \\
\hline \multicolumn{2}{|c|}{ TIR } & $\mathbf{1 6 \%}$ \\
\hline \multicolumn{2}{|c|}{ VAN } & $\mathbf{\$ 7 7 2 . 2 9 5}$ \\
\hline Tasa de interés para aval. \\
Financiera
\end{tabular}

El efecto de la variabilidad de las condiciones ambientales y ecológicas de una zona específica, es una realidad a la cual no escapa ninguna persona. Para el caso de los agricultores, su principal temor es la reducción en la producción al final de sus cultivos, y, por otra parte, el incremento del valor de los insumos. Entretanto, el 


\section{Utopía | \\ Working Papers \\ 2020}

principal temor del consumidor es la reducción de la oferta de los productos de la canasta familiar y como consecuencia, el aumento del precio de estos (Gerald et al., 2009).

Estudios realizados por Fondo Financiero de Proyectos de Desarrollo (FONADE) y por el Instituto de Hidrología, Meteorología y Estudios Ambientales (IDEAM), recopilados por Fernández (2013), los rendimientos de muchos cultivos podrían disminuir significativamente por las mayores temperaturas como consecuencia, por ejemplo, del estrés térmico e hídrico, del acortamiento de la estación de crecimiento y de la mayor presencia de plagas y enfermedades. Lo que se contrasta con la investigación realizada, en donde, por la variación irregular de la temperatura, pluviosidad y velocidades del viento, tuvo efecto en la producción del frijol, especialmente la alta pluviosidad en etapa de maduración de vainas.

\section{CONCLUSIONES}

El impacto que tienen las condiciones ecológicas de una zona tiene un efecto en la agricultura y por consiguiente en el bienestar de los agricultores y sus familias. En lo agrícola con respecto al rendimiento del cultivo, y como consecuencia precios, producción y consumo. En lo humano con la disminución en el consumo de nutrientes y malnutrición.

La acción de cultivar tiene una relación directa con la base de los recursos naturales, de manera particular con el agua, suelo y biodiversidad, fundamentales para su óptimo desarrollo. Sin embargo, el exceso de agua en etapas no deseadas ocasiona desventajas que conllevan a pérdidas económicas al productor.

La variedad de fríjol rojo cuarentano, cultivado en condiciones ambientales y ecológicas de El Yopal Casanare, entre los meses de julio a septiembre, la distancia entre plantas no tuvo una diferencia mínima significativa. Por lo tanto, para efectos de manejo agronómico, en las labores como fertilización edáfica se podría ampliar la densidad de siembra. 


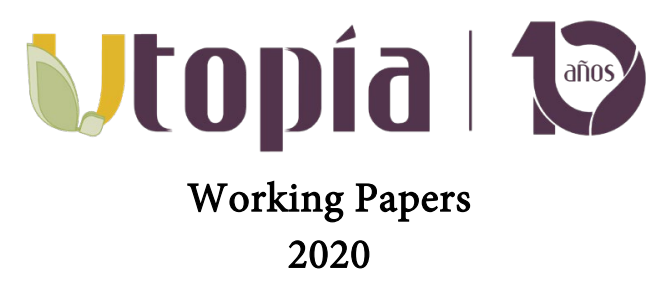

\section{BIBLIOGRAFÍA}

Astiasarán, I., Martínez, A. (2000). Alimentos Composición y Propiedades (Segunda ed.). Madrid, España: McGraw-Hill - Interamericana de España, S. A. U.

CIAT. (1983). Metodología para obtener semillas de calidad. Cali: Unidad de semillas del CIAT.

Consuegra, C. (11 de abril de 2018). Fríjol Rojo Cuarentano. Obtenido de Crepes y Waffles: https://crepesywaffles.com.co/blog/frijol-rojo-cuarentano

Fernández, M. (2013). Efectos del cambio climático en la producción y rendimiento de cultivos por sectores. Bogotá, D.C.: IDEAM.

Gerald, C., Rosegrant, M., Koo, J., Robertson, R., Sulser, T., Zhu, T., Lee, D. (2009). Cambio climático (El impacto en la agrícultura y los costos de adaptación). Washington, D:C: IFPRI.

Giral, A. (2016). ¿Qué pasa con el Agro en Colombia? Obtenido de https://www.gestiopolis.com/pasa-agrocolombia/

INATEC. (2003). Niveles y umbrales de daños económicos de las plagas.

López, H., Tosquy, O., Villar, B., Becerra, E., Ugalde, F. (2003). Adaptación, resistencia múltiple a enfermedades y tolerancia a suelos ácidos en genotipos de fríjol. Agronomía Mesoamericana, 151-2.

Mitidieri, M., Polack, L. (2012). Guía de monitoreo y reconocimiento de plagas, enfermedades y enemigos naturales de tomate y pimiento. Buenos Aires: INTA.

Nadal, S., Moreno, M., Cubero, J. (2004). Las leguminosas grano de la agricultura moderna. Madrid: MUNDIPRENSA.

Schoonhoven, A., Pastor, M. (1987). Evaluación Estándar para la Evaluación de Germoplasma de Fríjol. Cali: CIAT. 
https://doi.org/10.19052/wp.utopia.2020.3

\section{Utopía | \\ Working Papers \\ 2020}

Tovar, É. (7 de septiembre de 2013). Lo que tiene en jaque al agro colombiano. El Tiempo. Obtenido de https://www.eltiempo.com/archivo/documento/CMS-13052762

Tusquy, O., López, H., Acosta, J., Villar, B. (2014). Detection of black bean lines with adaptation in the humid tropics of southern Mexico. Revista Mexicana de Ciencias Agrícolas, 913. 